Journal of Advanced Research in Fluid Mechanics and Thermal Sciences

\title{
Experimental Study of Rigid and Flexible Tandem Wing for Micro Aerial Vehicle
}

\author{
Wan Mazlina Wan Mohamed ${ }^{1}$, Mohd Azmi Ismail2,, ${ }^{2}$, Muhammad Ridzwan Ramli ${ }^{3}$, Aliff Farhan \\ Mohd Yamin ${ }^{3}$, Koay Mei Hyie ${ }^{3}$, Hamid Yusoff ${ }^{3}$ Alam, Selangor, Malaysia.
2 School of Mechanical Engineering, Universiti Sains Malaysia, 14300, Nibong Tebal, Pulau Pinang, Malaysia \\ 1 Malaysia Institute of Transport (MITRANS), School of Mechanical Engineering, College of Engineering, Universiti Teknologi MARA, 40450 Shah \\ 3 Advanced Mechanics Research Group, Faculty of Mechanical Engineering, Universiti Teknologi MARA, Cawangan Pulau Pinang, 13500 Permatang \\ Pauh, Pulau Pinang, Malaysia
}

\section{ARTICLE INFO}

\section{Article history:}

Received 2 February 2021

Received in revised form 5 May 2021

Accepted 23 May 2021

Available online 5 August 2021

\section{Keywords:}

Lift Coefficient; Reynolds number; Angle of Attack

\section{ABSTRACT}

\section{Introduction}

Starting from the first aircraft invented by the Wright brothers in 1905, studies on airplane have become popular to this day. Many aircraft studies took place in 1973 when Boeing introduced computational fluid dynamic (CFD) as one of the aircraft research tools. Nowadays, many researchers have used CFD in aircraft studies to reduce the experimental cost as reported by Ismail and Wang [1], Sloan et al., [2] and Ives et al., [3] and Loutun et al., [4]. Ismail and Wang [1] employed CFD to study swirl flow of hot air for nacelle lip-skin application. A simulation study of bypass engine with fan nozzle exit area was studied by Sloan et al., [2], while Ives et al., [3] investigated the thermal

\footnotetext{
* Corresponding author.

E-mail address: azmi_meche@usm.my
}

https://doi.org/10.37934/arfmts.85.2.3343 
characteristic of bias acoustic liner (BAL) for aircraft application. Loutun et al., [4] employs CFD to study on the performance of various NACA airfoils.

However, the CFD studies require validation of experimental work to ensure its reliability as carried out by many previous researchers. Lee et al., [5] investigated aerodynamic performance of bias acoustic liner for nacelle application. Bahtia et al., [6] employed wind tunnel in China to study the boundary layer for different leading edge geometries. Aerodynamic wing study in wind tunnel test section was conducted by Hoang and Bui [7]. Although many aircraft studies have been conducted in the past, unmanned aerial vehicle (UAV) study has become increasingly popular over the past 15 years.

The success of UAVs in recent years has inspired more researchers to take an interest to perform in-depth analysis and improvement of similar reduced-size vehicle. Micro Aerial Vehicle (MAV) is a lightweight, autonomous air vehicle that is of interest to various fields, such as military and civilian applications [8]. The MAV is compact and can be automated or provided by a regulatory pilot offering an incentive to operate in confined area, making it suitable for public environmental activities, including observation. The term used by MAV is defined as an indoor air vehicle with a length of not more than $15 \mathrm{~cm}$, a gross drop weight (GTOW) of less than $200 \mathrm{~g}$ and a speed of $15 \mathrm{~m} / \mathrm{s}$ or less [8], [9]. The MAV can be divided into four types: fixed wing, rotary wing, bird-inspired ornithopter and entothopter, which are inspired from insects.

Fixed wing and ornithopter MAVs are ideal for high-capacity outdoor missions, rotary MAV is suitable for short-term outdoor operation, and for indoor missions, the entothopter MAV [10] type is the best. Propelled by characteristic of flyers, e.g., birds, bats and flying insects, the flapping wing, i.e., ornithopter and entothopter MAVs, is more powerful than the fixed wing and rotary wing MAVs as it has more moderate aerodynamic efficiency, particularly in low forward flight [10]. Pornsin-sirirak et al., [11] described three primary issues, namely (1) a reduced lift coefficient measures lower stacking limit; (2) increased drag coefficient implies high power input; (3) low angle of attack (AoA) output induces wing flow separation which results in low wing efficiency and manoeuvrability. They concluded that the low Reynolds flight efficiency for the fixed wing MAV was not as good as the flapping wing MAV flight.

Dragonfly is a remarkable creature due to its excellent flight characteristics, exceptionally agile, and capable of unexpectedly altering the course and pattern of flights. Adult dragonflies are capable of propelling themselves in six directions: up-down, forward, backward, turn left, and turn right [12]. In addition, dragonflies can be categorised into four different modes: counter-stroking staggered stroking, coordinated stroking and free flight $[12,13]$. Dragonflies are in slow or hovering mode for counter-stroking flight. In this mode, the wing is profiled by the forewing beats about $180^{\circ}$ from the phase (unparalleled motion) with the hindwings and efficiently generates a significant amount of lift. The phased stroking mode is performed by beating the hindwings $90^{\circ}$ ahead of the forewings [13]. This mode produces more thrust but lower lift than the counter-stroke. It is the ideal mode for fast forward flight. Synchronized stroking is where the hindwing and forewing beating together in phase (parallel frequency motion) has maximized the lift acceleration [14]. It is very useful for tandem wing insects such as dragonflies, and they should accelerate immediate flight direction change. The final flight style for dragonflies is to glide mode. In this mode, the wings are simply stretched without flapping [13] or are stationary. Interestingly, the wings of flying insects usually account for about $2 \%$ of their overall bodyweight [15]. This has prompted many researchers to perform studies on new type of unmanned medium-sized aircraft known as biomimetic micro air vehicle (BMAV). BMAV has great potential to be applied to the next generation of UAV due to its compact size and lightweight resembling its natural counterpart [16]. 
Wing arrangement plays an important role either in single or tandem wing flapping natural flyers. Studies of the time unsteady aerodynamic system in tandem flapping-wing insects are more complex than a single wing. According to the literature, tandem flapping wing increases the generation of lift forces and acceleration [12]. The relationship between the forewing and hindwing is defined based on the interaction of hindwing with forewing wake flow as an important dependency of the tandem wing flapping. In the case of dragonfly wings, the wings are only appropriate for single wing movement with a certain angle of attack during flapping. It also fits the phase angle during the flapping of both tandem wing pairs at random $[12,17]$. In tandem wing flying mode, there are two pairs of functional wings, namely forewings and hindwings. In the early stage, the MAV production, research and experiment have focused on a single flapping wing to study the characteristics which controlled the aerodynamic performance. Therefore, it is proposed to create a working MAV flying mechanism. The subsequent stage of MAV research is the focus on tandem wing configuration. The objective is to observe the aerodynamic efficiency of tandem flapping insects, especially dragonflies.

The MAV studies have been performed by numerous researchers. According to the existing literature, most of the MAV studies have employed rigid wing skin. However, actual insect such as dragonfly wing membranes are not entirely rigid but more flexible. Rigid wing skin for single and tandem arrangement studies have been conducted by many researchers. Nevertheless, a flexible wing skin for dragonfly imitation design on tandem wing arrangements has not been adequately studied, especially at frequency of $11 \mathrm{~Hz}$, and various angles of attack. Thus, this paper discusses the lift coefficient profile of wing dragonfly imitation MAV at frequency of $11 \mathrm{~Hz}$, three different Reynolds numbers and various AoA for flexible wing skin tandem arrangement. Subsequently, comparison between lift coefficients for both rigid and flexible wing skins on tandem arrangement is elaborated at the end of the result and discussion section.

\section{Methodology}

\subsection{MAV Model Development}

The design of the MAV structure studied has followed the MAV design by H. Takashashi [18], which has also been used by Deng et al., [19]. Microelectromechanical device (MEMS) with pressure sensor was employed to move the forewing and hindwing. The MAV geometric design was developed by SolidWorks, and fabricated by Fused Deposition Printing method. Polylactic acid was used as MAV structure material as it has higher material strength compared to other materials from 3D printer. Figure 1 illustrates the 3D geometric modelling of the MAV studied.

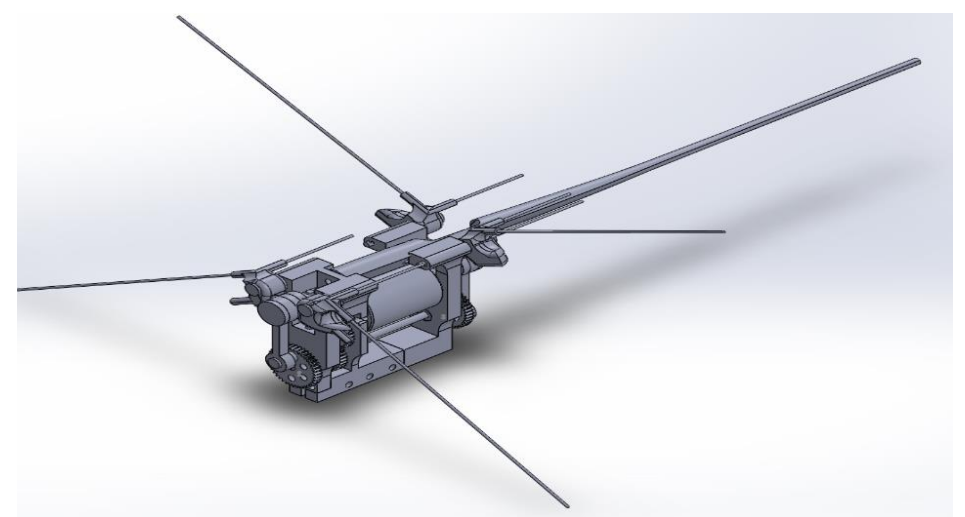

Fig. 1. Schematic drawing of MAV in this study 
As shown in Figure 1, the design of MAV did not $100 \%$ imitate the dragonfly. Only wing structure, size and flapping movement of the MAV studied were inspired by dragonfly. Two types of materials were used in flapping wing skin, namely laminating film for rigid wing skin and latex for flexible wing skin. Figure 2 and 3 show the dimension of the wing and the MAV assembly in this study. The information of mass, strength and dimension of the MAV studied is summarised in Table 1 . The wing span and cord length were $12 \mathrm{~cm}$ and $4 \mathrm{~cm}$, respectively. The thickness of the wing laminating film was $0.37 \mathrm{~mm}$, which was $0.24 \mathrm{~mm}$ thicker than the latex flexible wing. Although the elastic modulus (0.71 MPa) and specific thickness (1.6) of the rigid wing were higher than those of flexible wing, the mass of flexible wing $(62 \mathrm{mg}$ ) was lighter than the mass rigid wing $(130 \mathrm{mg})$. The overall masses of MAV with rigid body and flexible body were $6.8 \mathrm{~g}$ and $3.4 \mathrm{~g}$, respectively.

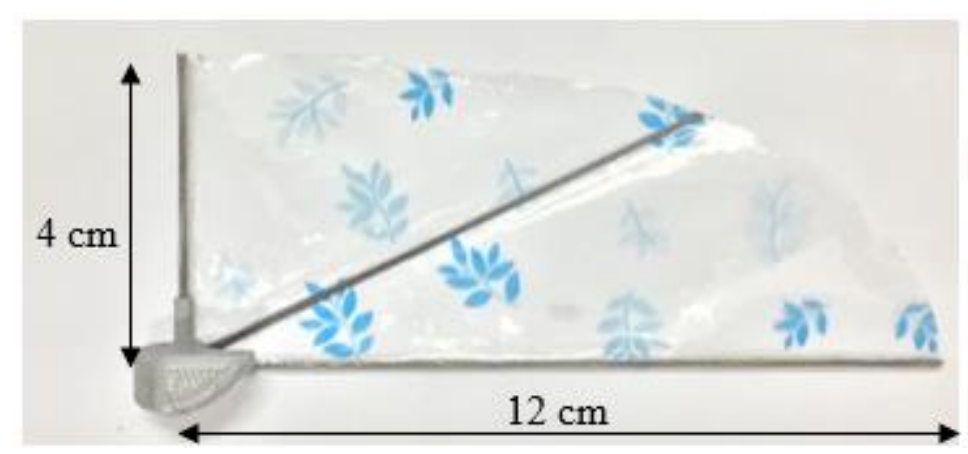

Fig. 2. Dimension of wing

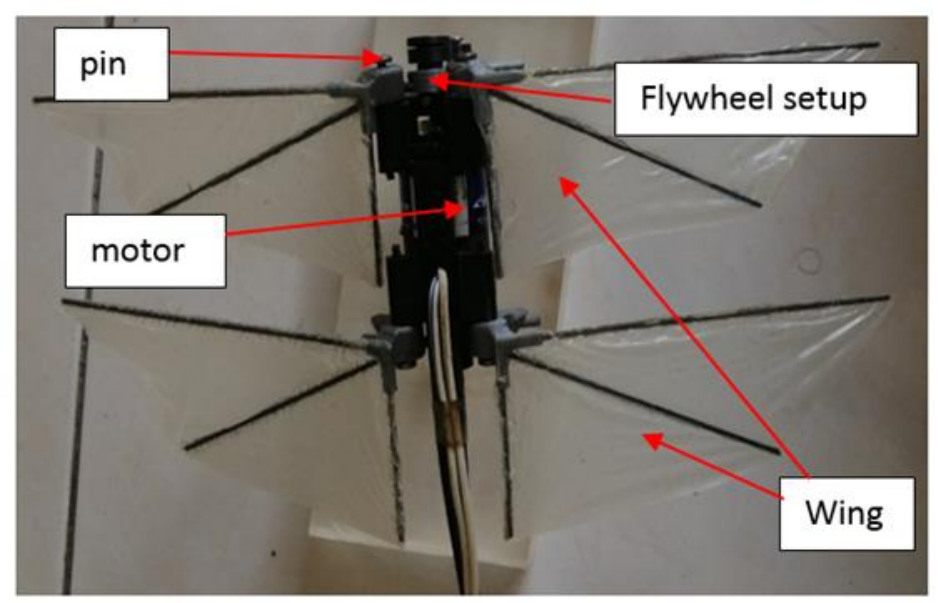

Fig. 3. Assembly of MAV in this study 


\section{Table 1}

The Details of each part of rigid and flexible tandem flapping wing testbed

\begin{tabular}{lll}
\hline Part of Rigid Flapping & $\begin{array}{l}\text { Dimension of } \\
\text { Rigid Membrane }\end{array}$ & $\begin{array}{l}\text { Dimension of } \\
\text { Flexible Membrane }\end{array}$ \\
\hline Wingspan & $120 \mathrm{~mm}$ & $120 \mathrm{~mm}$ \\
Wing Chord Length & $40 \mathrm{~mm}$ & $40 \mathrm{~mm}$ \\
Constant Wing Thickness & $0.37 \mathrm{~mm}$ & $0.13 \mathrm{~mm}$ \\
Distance between & $10 \mathrm{~mm}$ & $10 \mathrm{~mm}$ \\
Forewing and Hindwing & & \\
Leading Edge Support & $1 \mathrm{~mm}$ & $1 \mathrm{~mm}$ \\
Body Side Support & $1 \mathrm{~mm}$ & $1 \mathrm{~mm}$ \\
Elastic Modulus & $0.71 \mathrm{MPa}$ & $0.64 \mathrm{MPa}$ \\
Specific Stiffness & 1.6 & 1 \\
Wing Weight & $130 \mathrm{mg}$ & $62 \mathrm{mg}$ \\
Total Weight & $6.8 \mathrm{~g}$ & $3.4 \mathrm{~g}$ \\
\hline
\end{tabular}

\subsection{Experimental Setup}

Experimental tests were conducted in mini wind tunnel at Universiti Sains Malaysia. The experimental setup consisted of wind tunnel, balancing unit, data acquisition (DAQ) and computer. Wind tunnel was used to supply the air velocity to the MAV. Balancing unit and DAQ were used to measure the lift force. All recorded data were saved in the computer. Figure 4 illustrates the schematic diagram of the experimental setup.

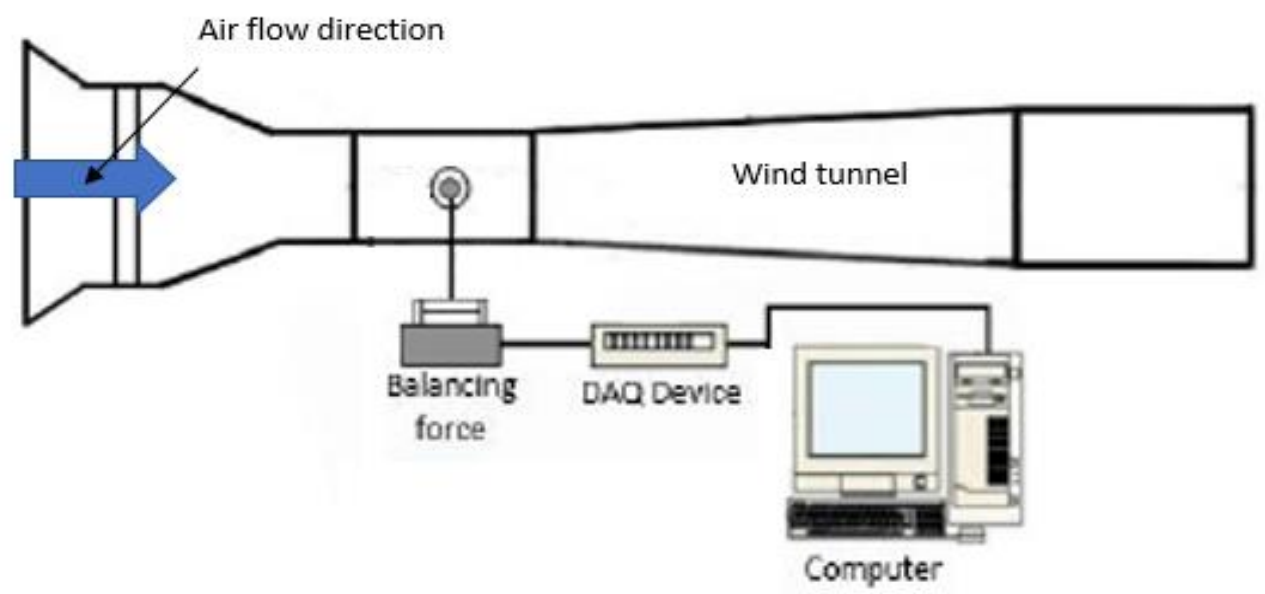

Fig. 4. Schematic diagram of lift force at wind tunnel setup

The MAV was installed in the test section of $30 \mathrm{~cm} \times 30 \mathrm{~cm} \times 50 \mathrm{~cm}$ wind tunnel as illustrated in Figure 5. The MAV was connected to the balancing unit using thin and light aluminium rod. As shown in Figure 5 , the $A O A$ of MAV was controlled by $A o A$ transducer with a precision of $1^{\circ}$. Pitot tube was used to measure the dynamic pressure and wind tunnel air velocity. DC power supply was employed to supply electric current to the MAV motor to flap the MAV wing. The balancing unit consisting of stain gage was used to measure the lift force of the MAV studied. 

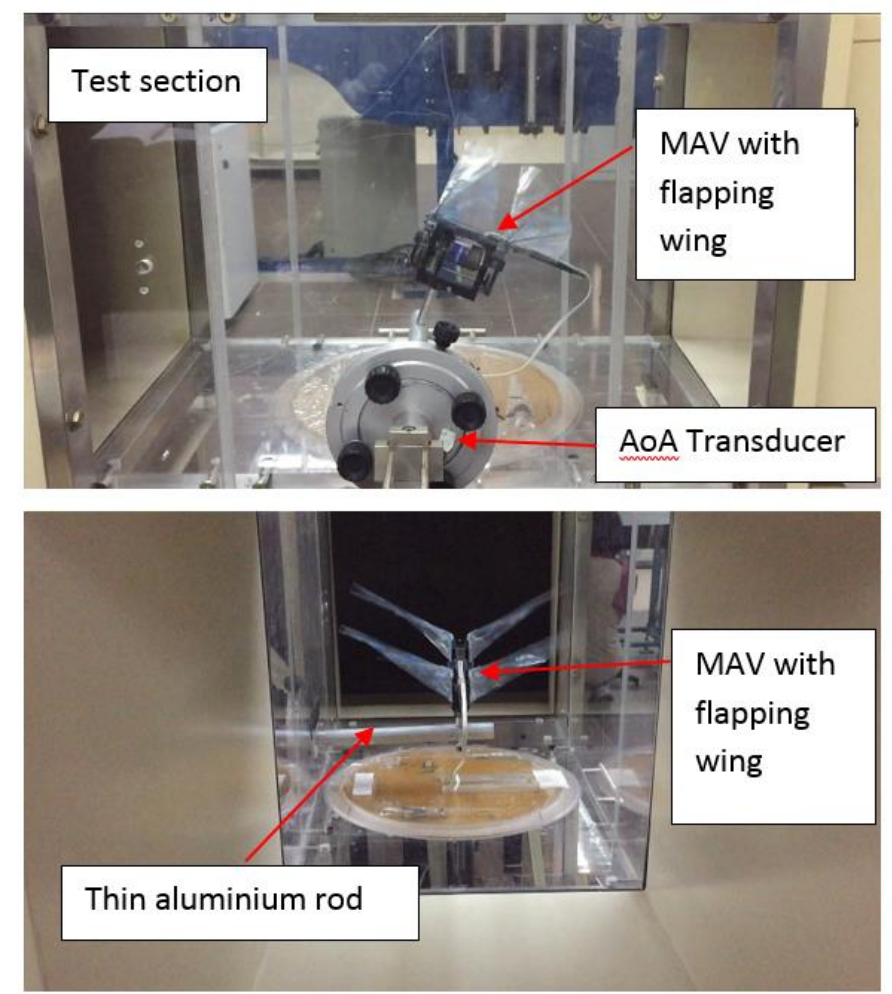

Fig. 5. Side view (above) and rear view (below) of the flapping mechanism in the wind tunnel

In this study, DAQ was used to convert analogue signal from balancing unit into digital data. The digital data were then recorded and saved in the computer. The DAQ model Kyowa PCD-300A has four channels, and it was connected to computer by cable. Before the experimental test commenced, the balancing unit with DAQ was calibrated. Lab View code was utilised to convert digital signal to be saved in Microsoft Excel spreadsheet.

\subsection{Operating Condition}

The operating conditions involved in this study were similar to the previous study by Ismail et al., [12]. Based on their results, the highest frequency was produced at wing flapping frequency of 11 $\mathrm{Hz}$. Therefore, the wing flapping frequency selected for this study was $11 \mathrm{~Hz}$. Three different air velocities of 5,7 and $9 \mathrm{~m} / \mathrm{s}$, and six different $A \circ A^{\prime} \mathrm{s}$ of $0^{\circ}, 10^{\circ}, 20^{\circ}, 30^{\circ}, 40^{\circ}, 50^{\circ}$, and $60^{\circ}$ were investigated in this study. The Reynolds number $(\mathrm{Re})$ and the lift coefficient $\left(C_{L}\right)$ were calculated from Eq. (1) [20] and Eq. (2) [9], respectively. All information on operating conditions is summarised in Table 2.

Table 2

Operating conditions of rigid and flexible membrane of tandem flapping wing

\begin{tabular}{lll}
\hline Part of Rigid Flapping & $\begin{array}{l}\text { Dimension of Rigid } \\
\text { Membrane }\end{array}$ & $\begin{array}{l}\text { Dimension of } \\
\text { Flexible Membrane }\end{array}$ \\
\hline Flapping frequencies, $\mathrm{Hz}$ & 11.0 & 11.0 \\
Velocity, $\mathrm{m} / \mathrm{s}$ & $5.0,7.0$, and 9.0 & $5.0,7.0$ and 9.0 \\
AoA, $\left(^{\circ}\right)$ & $0-50$ & $0-50$ \\
Reynolds number & 14000,19000, and & 14000,19000 and \\
& 24000 & 24000 \\
\hline
\end{tabular}


$R e=\frac{U_{\infty} D_{H}}{v}$

where, $v$ is the kinematic viscosity of air, $D_{H}$ is the hydraulic diameter and $U_{\infty}$ is wind tunnel air velocity. In this study, $D_{H}$ represented the mean chord wing $=0.04 \mathrm{~m}$,

$C_{L}=\frac{2 F_{L}}{\rho x U_{\infty} x A}$

where, $F_{L}$ is the lift force, $\rho$ is the air density and $A$ is the projection of wing surface area.

\section{Results and Discussion}

\subsection{Effect of Rigid Tandem Wing Skin as Function of AoA and Re}

Figure 6 shows the effect of $A \circ A$ on $C_{L}$ for rigid wing at flapping frequency of $11 \mathrm{~Hz}$. It can be seen that $C_{L}$ was proportional to $A O A$. At $A O A$ of $0^{\circ}$, the $C_{L}$ for all Re values in the figure were negative. Subsequently, a monotone increase with respect to $A o A$ for all Re values before the peak value at $A \circ A$ of $50^{\circ}$ for rigid tandem wing skin was achieved. Re of 14000 produced an increase in $C_{L}$ of 10 with $A O A$ ranging from $0^{\circ}$ to $50^{\circ}$. The highest $C_{L}$ was obtained at $R e$ of 14000 , while the lowest $C_{L}$ against $A O A$ was obtained at $R e$ of 24000 . With $A o A$ from $0^{\circ}$ to $50^{\circ}$, total increment of $C_{L}$ for $R e$ of 14000 was similar to $R e$ of 24000 . However, at $R e$ of 19000 , the total increment of $C_{L} 20 \%$ lower than the Re of 14000 and 24000. Based on this trend, it can be concluded that high Re produced low $C_{L}$. It is expected that the lift is created by the formation of the vortex at the top of the wing. When AoA reached $50^{\circ}$, based on graph projection, it was predicted that the peak value of rigid tandem wing skin indicated that $A O A$ of $50^{\circ}$ has stalled.

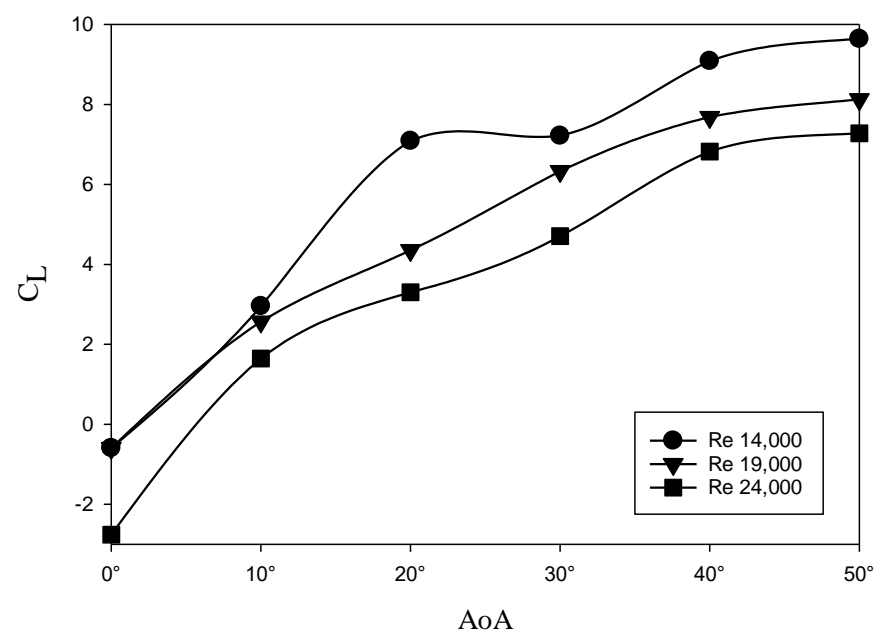

Fig. 6. Lift coefficient against angle of attack for rigid wing skin

\subsection{The Effect of Flexible Tandem Wing Skin as Function of AoA and Re}

Figure 7 shows $C_{L}$ profile for flexible tandem wing against $A o A$ for three $R e$ values from 14000 to 24000 at $11 \mathrm{~Hz}$ frequency. It was found that the $C_{L}$ increased with increasing $A O A$ for all Re values. At $R e$ of 19000, the $C_{L}$ increased from 1 to 6 as $A o A$ increased from $0^{\circ}$ to $50^{\circ}$. The findings of the experimental analysis revealed that the flight at lower Re provided higher $C_{L}$ for the flexible tandem 
wing skin compared to higher $R e$, similar to the case of rigid wing. As shown in Figure 7, $R e$ of 14000 showed the highest $C_{L}$, followed by $R e$ of 19000 and 24000 . It can be summarised that $C_{L}$ was higher for lower Re compared to higher Re. For example, at $A \circ A$ of $50^{\circ}, C_{L}$ at $R e$ of 14000 was $80 \%$ higher than that of $R e$ of 24000 . This may be attributed to the lift productivity at high $R e$ which decreased the reduction of the effective $A o A$. During the flapping flight at high $R e$, the hindwing vortex deformed the trailing edge of the hindwing which inclined to reduce the effective $A O A$ and reduced the lift efficiency. Figure 7 shows an increasing trend as $A o A$ increased. It was predicted that unlike rigid wing, $C_{L}$ will increase even when the $A \circ A$ exceeds $50^{\circ}$.

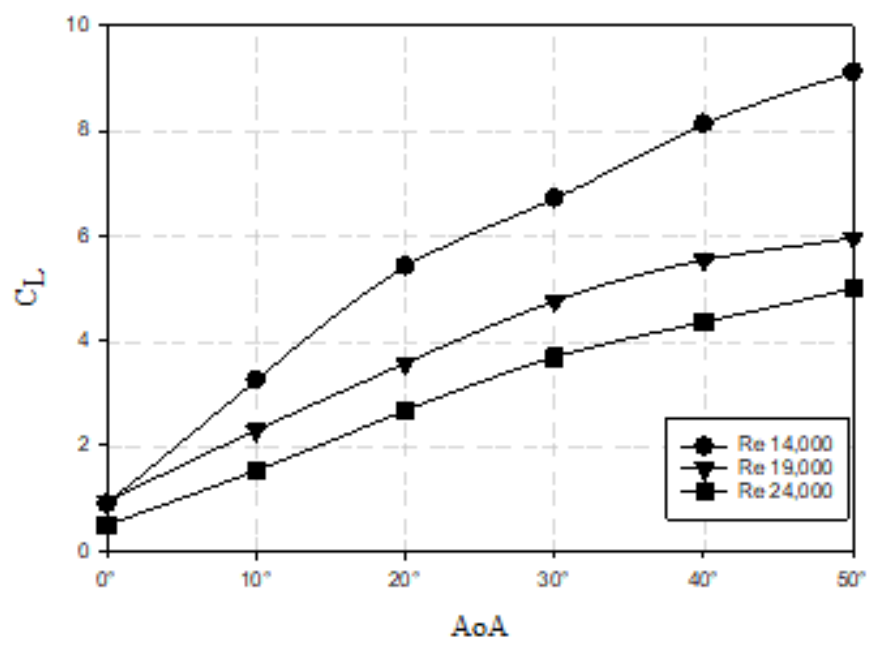

Fig. 7. Lift coefficient against $A o A$ for flexible wing skin

\subsection{Comparison between Rigid and Flexible Tandem Wing Skin as Function of AoA and Re}

Figure 8,9 , and 10 display the comparison of $C_{L}$ for rigid wing and flexible wing at $A \circ A$ from $0^{\circ}$ to $50^{\circ}$ for $R e$ of $14000, R e$ of 19000 , and $R e$ of 24000 , respectively. Figure 8 shows that $C_{L}$ for flexible wing skin demonstrated positive value at $A O A$ of $0^{\circ}$, similar to Figure 9 and 10 . However, Figure 8, 9, and 10 showed negative $C_{L}$ values at $A O A$ of $0^{\circ}$. This indicated that the flexible wing skin improved aerodynamic performance at low $A o A$. The highest $C_{L}$ at $A o A$ of $0^{\circ}$ for flexible wing skin is shown in Figure 8. Thus, it concluded that lower Re produced higher $C_{L}$.

Referring to the figures, the unexpected consequence of low AoA for all the flight speeds was that $C_{L}$ for flexible tandem wing skin offered more improvement in lift generation than the rigid tandem wing skin. The lift coefficient was higher at lower Re due to changes in AoA caused by the formation of higher separation vortexes, where lower airflow rate was used. A previous study reported an improvement in lift performance by the flexibility of the membrane wing skin at low Re, while at high $\mathrm{Re}$, the patterns were opposite where most rigid membrane displayed the weakest lift generation [8, 10]. Yussof [9] in his research also stated that at low Re, the tandem flapping-wing provided a better lift generation.

The effect of the wing membrane on the tandem flapping-wing at high $A o A$ was also observed in Figure 8,9 and 10. As shown in Figure 10, at $A \circ A$ of $50^{\circ}$, the $C_{L}$ of flexible wing was approximately $30 \%$ lower than that of rigid wing. However, at low $R e$ as shown in Figure 8, the $C_{L}$ for flexible wing was only approximately $5 \%$ lower than that of rigid wing skin at $A O A$ of $50^{\circ}$. The findings are not expected as the flexible wing produced lower $C_{L}$ than the rigid wing. The potential reason could be due to the turbulent airflow hit the wing at higher $R e$ and combined with the aid of vibration disruption, thus creating a prematurely smaller preparation vortex. A delayed stall occurred as the 
downwash increase which simultaneously decreased the performance of $A \circ A$, causing increased deformation at the trailing edge, which in turn reduced the lift. This has obviously demonstrated that the rigid tandem wing skin has a better performance compared to the flexible tandem wing skin, while $A o A$ increased at appropriate Re level for lift coefficient generation performance.

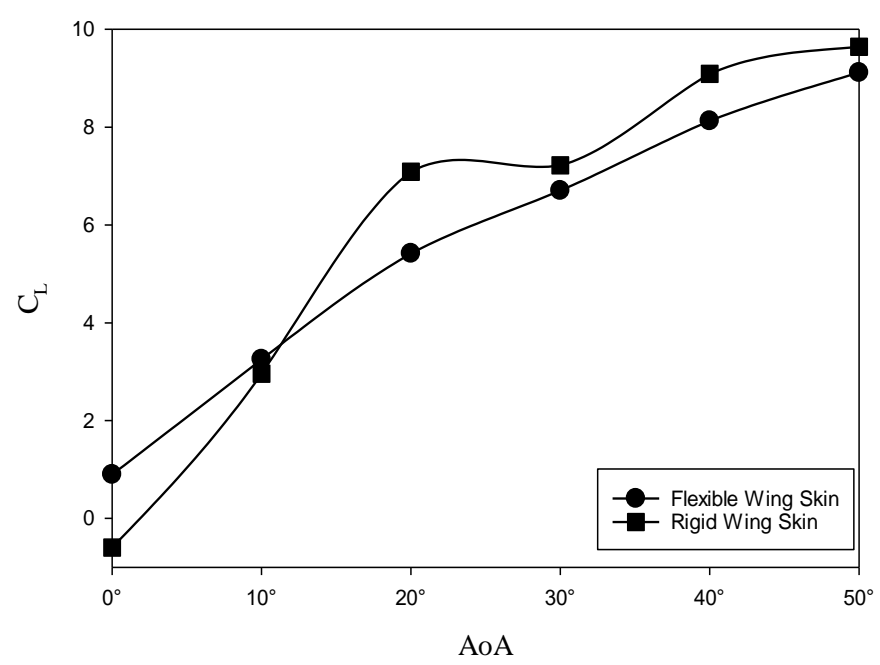

Fig. 8. The comparison of $C_{L}$ for rigid and flexible wings skin at $R e$ of 14000

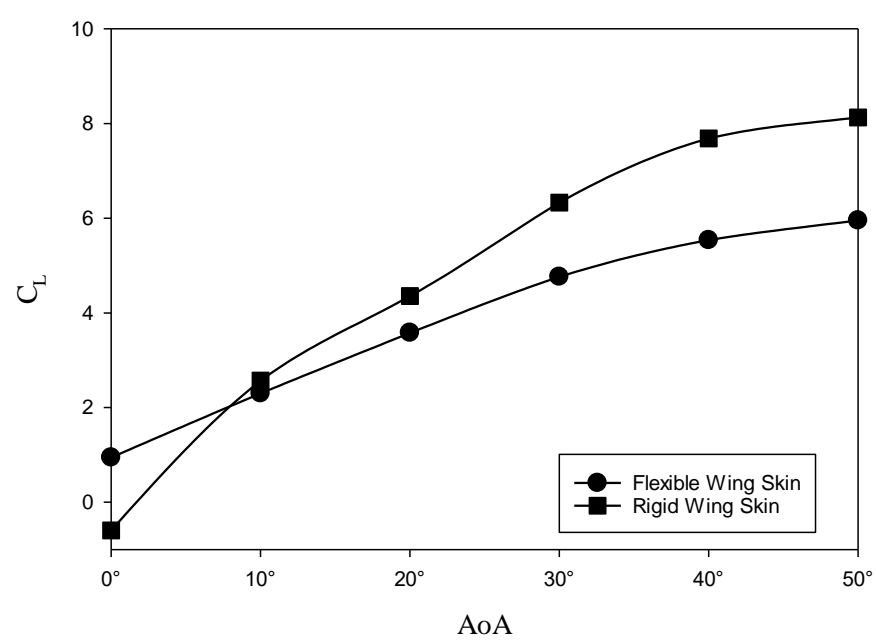

Fig. 9. The comparison of $C_{L}$ for rigid and flexible wings skin at Re of 19000 


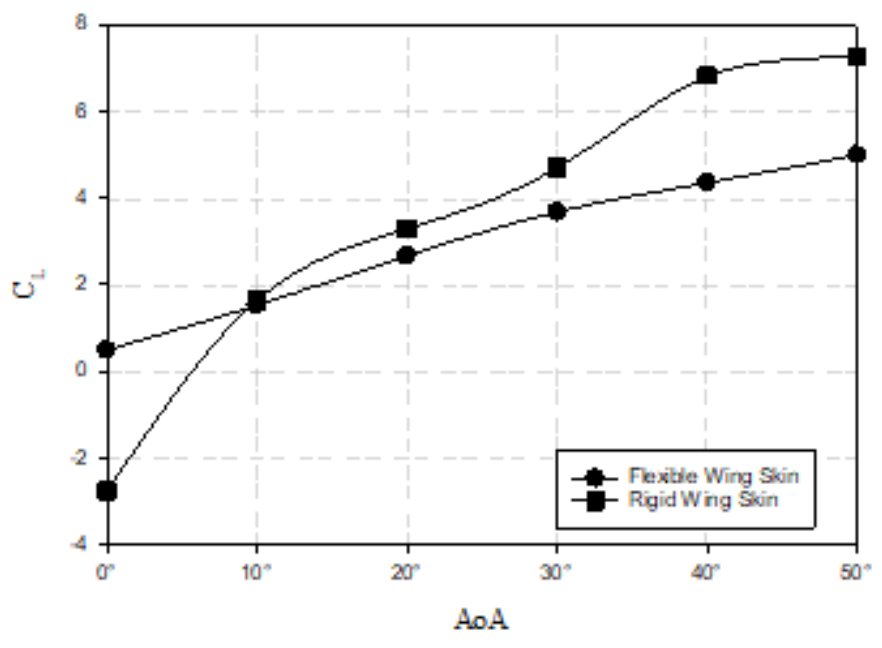

Fig. 10. The comparison of $C_{L}$ for rigid and flexible wings skin at $R e$ of 24000

\section{Conclusions}

The effects of rigid and flexible wing skins on the aerodynamic performance were studied at wing flapping frequency of $11 \mathrm{~Hz}, \operatorname{Re}$ of 14000,19000 and 24000 , and $A o A$ between $0^{\circ}$ and $50^{\circ}$. The novelty of this research was to focus on the same mechanism for rigid and flexible tandem wing skins. The conclusions of this study are as follows

i. Lower Re produced higher $C_{L}$ than higher Re. For flexible wing study, Re of 14000 produced $100 \% C_{L}$ than $\operatorname{Re}$ of 24000.

ii. At low $A \circ A$, the flexible tandem wing skin provided higher lift performance compared to the rigid tandem wing skin. At $R e$ of 24000 , flexible wing has $C_{L}$ approximately 2.5 higher than the rigid wing. The flexible wing skin also showed decrease in the effect of camber at low Re to achieve better lift performance.

iii. At high $A \circ A$, high trailing edge deformation of the flexible tandem wing skin induced the effective $A o A$ to decrease, thereby reducing lift efficiency relative to rigid wing skin even at low Re.

\section{Acknowledgement}

The authors would like to acknowledge the financial support from Malaysia Institute of Transport (MITRANS) and Universiti Teknologi MARA, Cawangan Pulau Pinang.

\section{References}

[1] Ismail, Mohd Azmi, and Jian Wang. "Effect of nozzle rotation angles and sizes on thermal characteristic of swirl antiicing." Journal of Mechanical Science and Technology 32, no. 9 (2018): 4485-4493. https://doi.org/10.1007/s12206018-0845-x

[2] Sloan, B., J. Wang, Stephen Spence, S. Raghunathan, and D. Riordan. "Aerodynamic performance of a bypass engine with fan nozzle exit area change by warped chevrons." Proceedings of the Institution of Mechanical Engineers, Part G: Journal of Aerospace Engineering 224, no. 6 (2010): 731-743. https://doi.org/10.1243/09544100JAERO529

[3] Ives, Anthony O., Jian Wang, Srinivasan Raghunathan, and Patrick Sloan. "Heat transfer through a single hole bias flow acoustic liner." Journal of thermophysics and heat transfer 25, no. 3 (2011): 409-423. https://doi.org/10.2514/1.T3637

[4] Loutun, Mark Jason Thomas, Djamal Hissein Didane, Mohd Faizal Mohideen Batcha, Kamil Abdullah, Mas Fawzi Mohd Ali, Akmal Nizam Mohammed, and Lukmon Owolabi Afolabi. "2D CFD Simulation Study on the Performance of Various NACA Airfoils." CFD Letters 13, no. 4 (2021): 38-50. https://doi.org/10.37934/cfdl.13.4.3850 
[5] Khai, Lee Chern, Mohd Azmi Ismail, Qummare Azam, and Nurul Musfirah Mazlan. "Experimental study on aerodynamic performance of nacelle lip-skin bias flow." Journal of Mechanical Science \& Technology 34, no. 4 (2020). https://doi.org/10.1007/s12206-020-0323-0

[6] Bhatia, Dinesh, Guang Jun Yang, Jing Sun, and Jian Wang. "Effect of Leading Edge Geometry on Boundary Layer Transition-An Experimental Approach." In Applied Mechanics and Materials, vol. 590, pp. 53-57. Trans Tech Publications Ltd, 2014. https://doi.org/10.4028/www.scientific.net/AMM.590.53

[7] Hoang, Ngoc TB, and Binh V. Bui. "Investigation of wind tunnel wall effect and wing-fuselage interference regarding the prediction of wing aerodynamics and its influence on the horizontal tail." Journal of Mechanical Science and Technology 33, no. 6 (2019): 2737-2746. https://doi.org/10.1007/s12206-019-0520-x

[8] Lian, Yongsheng, Timothy Broering, Kyle Hord, and Russell Prater. "The characterization of tandem and corrugated wings." Progress in Aerospace Sciences 65 (2014): 41-69. https://doi.org/10.1016/j.paerosci.2013.08.001

[9] Yusoff, H., M. Z. Abdullah, M. Abdul Mujeebu, and K. A. Ahmad. "Effect of skin flexibility on aerodynamic performance of flexible skin flapping wings for micro air vehicles." Experimental Techniques 39, no. 1 (2015): 1120. https://doi.org/10.1111/ext.12004

[10] Ratti, Jayant, and George Vachtsevanos. "A biologically-inspired micro aerial vehicle." Journal of Intelligent \& Robotic Systems 60, no. 1 (2010): 153-178. https://doi.org/10.1007/s10846-010-9415-x

[11] Pornsin-Sirirak, T. Nick, Y. C. Tai, H. Nassef, and C. M. Ho. "Titanium-alloy MEMS wing technology for a micro aerial vehicle application." Sensors and Actuators A: Physical 89, no. 1-2 (2001): 95-103. https://doi.org/10.1016/S09244247(00)00527-6

[12] Ismail, N. I., H. Yusoff, S. Budin, and A. F. M. Yamin. "An experimental mechanism of a tandem flapping wing for micro aerial vehicle." In Journal of Physics: Conference Series, vol. 1349, no. 1, p. 012014. IOP Publishing, 2019. https://doi.org/10.1088/1742-6596/1349/1/012014

[13] Salami, Erfan, Thomas A. Ward, Elham Montazer, and Nik Nazri Nik Ghazali. "A review of aerodynamic studies on dragonfly flight." Proceedings of the Institution of Mechanical Engineers, Part C: Journal of Mechanical Engineering Science 233, no. 18 (2019): 6519-6537. https://doi.org/10.1177/0954406219861133

[14] Chalia, Sonia, and Manish Kumar Bharti. "A Review on Aerodynamics of Flapping Wings." International Research Journal of Engineering and Technology 3, no. 3, (2016): 739-743.

[15] Salami, Erfan, E. Montazer, T. A. Ward, and P. B. Ganesan. "Nano-mechanical properties and structural of a 3Dprinted biodegradable biomimetic micro air vehicle wing." In IOP Conference Series: Materials Science and Engineering, vol. 210, no. 1, p. 012073. IOP Publishing, 2017. https://doi.org/10.1088/1757-899X/210/1/012073

[16] Miller, Laura A., and Charles S. Peskin. "Flexible clap and fling in tiny insect flight." Journal of Experimental Biology 212, no. 19 (2009): 3076-3090. https://doi.org/10.1242/jeb.028662

[17] Hefler, Csaba, Tim Marco Corti, and Huihe Qiu. "Aerodynamic Analysis of a Bioinspired Multilayer Flexible Wing." In Proceedings of the 2nd World Congress on Mechanical, Chemical, and Material Engineering (MCM'16). 2016. https://doi.org/10.11159/htff16.137

[18] Takahashi, Hidetoshi, Alice Concordel, Jamie Paik, and Isao Shimoyama. "The effect of the phase angle between the forewing and hindwing on the aerodynamic performance of a dragonfly-type ornithopter." Aerospace 3, no. 1 (2016): 4. https://doi.org/10.3390/aerospace3010004

[19] Deng, Shuanghou, Jun Wang, and Hanru Liu. "Experimental study of a bio-inspired flapping wing MAV by means of force and PIV measurements." Aerospace Science and Technology 94 (2019): 105382. https://doi.org/10.1016/i.ast.2019.105382

[20] Ismail, Mohd Azmi, R. H. Z. Yu, M. I. Ramdan, and K. H. Yu. "EXPERIMENTAL STUDY OF SYNTHETIC JET PERFORMANCE IN COMBINED CONVECTION HEAT TRANSFER." Heat Transfer Research 52, no. 1 (2021). 1-15. https://doi.org/10.1615/HeatTransRes.2020033493 